\title{
Presentación
}

\section{Sociodemografía del envejecimiento poblacional en México}

Rebeca Wong*

El envejecimiento poblacional es uno de los fenómenos demográficos de mayor importancia para México y para el resto del mundo en la primera mitad del siglo XXI. Como se ha discutido en varias publicaciones en los últimos años, éste representa un cambio radical en la estructura por edad de la población a favor de las edades medias y avanzadas, que se dio como resultado de la caída de la mortalidad y de la fecundidad por un periodo largo y de manera sostenida en la segunda mitad del siglo XX (véase, por ejemplo, Conapo, 1999; Kinsella, 2000).

México cuenta con una "ventana de oportunidad" de aproximadamente dos a tres décadas debido a que la tasa de dependencia demográfica primero bajará para después subir cuando se acelere el envejecimiento (Hill et al., 1999). Este periodo de oportunidad, con baja tasa de dependencia, podría usarse para desarrollar los mercados financieros, reformar los sistemas fiscales, de pensiones y de salud, y aumentar el capital social con el que pueda contar la población de edad media y avanzada en el futuro. Sin embargo, el desarrollo de tales mercados puede tardar varios lustros y un nuevo plan de seguridad social llevaría aproximadamente tres décadas para madurar. Desde el punto de vista de la oportunidad, la comunidad científica demográfica, y en particular los estudiosos del envejecimiento poblacional, tienen el reto de hacer oír su voz para que durante este periodo se aumente el conocimiento que permita planear para el futuro y contribuir al diseño de nuevas políticas sociales, económicas y de población, al mismo tiempo que se afronten las limitaciones actuales de desigualdad social y económica en el país.

En este volumen se presentan trabajos en sociodemografía del envejecimiento realizados por investigadores de múltiples disciplinas de las ciencias sociales (demógrafos formales, demógrafos sociales y económicos, antropólogos, y de políticas públicas), reconociendo que el envejecimiento poblacional se está estudiando desde una perspectiva multidisciplinaria. Además creemos que los estudios sobre el

* Georgetown University, Center for Population Research. Correo electronico: rwong@georgetown.edu 
fenómeno del envejecimiento se enriquecen mediante el distinto nivel de conocimiento que se adquiere a través de métodos tan to cualitativos como cuantitativos, por lo que se incluyen artículos que presentan ambas perspectivas. Los artículos incluidos fueron seleccionados de trabajos recientes recibidos para su publicación en la revista Estudios Demográficos y Urbanos, así como de los que se presentaron en las Reuniones Nacionales de Demografía en las mesas sobre Envejecimiento, en México en agosto de 2000.

Los cambios en la estructura por edad de la población en los países desarrollados se dieron acompañados de una transformación social y económica, dando paso a un nuevo sistema que se generaliza en forma global: la nueva era de la información (Giddens, 1990). Es claro que aún no sabemos lo que este nuevo sistema económico implicará para las relaciones sociales y el comportamiento demográfico de la población. Alejandro Canales Cerón, en su trabajo sobre la era de la información y el envejecimiento demográfico, concluye que es importante hacer cambios al discurso demográfico en México. El artículo presenta aspectos de las relaciones entre la población y el sistema económico de producción, ambos en profunda transformación actual o inminente. La discusión de estos temas puede ayudar a reformular el discurso de las políticas de población ya que se está transitando de la era industrial a la era de la información con cambios en las relaciones sociales, y particularmente en el mercado de trabajo, lo cual tiene manifestaciones sin precedente histórico en la dinámica demográfica. Como lo indica el autor, el desafio de la demografía es pensar en la población ya no en términos de su crecimiento en números sino de las relaciones entre sus individuos y las instituciones, así como entre la especie y la naturaleza. Cuando pensamos en la velocidad en que están ocurriendo cambios en la era de la información, donde se transforma la manera de procrear, se aumenta la propensión a las adopciones de niños a escala mundial, y se cambian incluso, las formas de migrar, de obtener ingresos o adquirir capital humano debido a la velocidad con que se intercambia y se adquiere información, estamos enfrentando un fenómeno demográfico distinto en el que el discurso poblacional necesita transformarse. Dentro de esta reflexión, el autor, más que ofrecer respuestas o predicciones definitivas, presenta una serie de preguntas que nos obligan a pensar en otras formas de realizar investigaciones y estudios que alimenten un nuevo discurso demográfico.

Por ejemplo, el autor pregunta: ¿cómo se irá valorando de manera distinta a la juventud y a la vejez en un marco de producción eco- 
nómica que ya no requiere tanto del trabajo físico y ante el envejecimiento poblacional?, ¿cómo irá cambiando el papel que juega el anciano en nuestra sociedad?, ¿qué tensiones se darán entre los intercambios generacionales, tanto en forma personal o individual, como a través de las instituciones? Sin decirlo con estas palabras, el artículo se puede entender como un llamado a los demógrafos a abrir la reflexión hacia los aportes disciplinarios que contribuyan a enriquecer el conocimiento colectivo en las áreas que les competen. Además, con las preguntas que plantea, el artículo ilustra cómo el estudio sociodemográfico del envejecimiento tiene que ir más allá de estudios sobre poblaciones de edades media y avanzada. El envejecimiento poblacional afecta a todos los grupos de edad, y por ello tiene impactos en múltiples áreas de estudio. Se podría agregar que en el espíritu de la nueva era de la información, los resultados de los estudios realizados dentro del ambiente académico deben ser remitidos también a los encargados de diseñar políticas públicas, de manera oportuna y con un lenguaje accesible.

A partir del segundo artículo los trabajos tratan temas que se relacionan con la satisfacción de las necesidades de bienestar de la población en edad avanzada. Su salud es sin lugar a dudas la dimensión más importante de su bienestar, y este volumen incluye varios artículos que se refieren a ella. En el artículo de Rebeca Wong y María Aysa Lastra se presenta un marco conceptual para hacer estudios sociodemográficos de la salud a escala poblacional. Se hace énfasis en que se requiere contar con bases de datos con propósitos múltiples donde se cubran no sólo las características demográficas y socioeconómicas de los individuos y se relacionen con aspectos acerca de su salud y el uso de servicios para mantenerla, sino también que den cuenta del papel que juegan sus redes familiares de apoyo y su historia laboral y de migración, de modo tal que permitan entender los cambios que se van dando en la salud en las edades medias y avanzadas. Esta propuesta refuerza la necesidad de conducir estudios longitudinales, sobre todo cuando se trata de estudiar la dinámica del envejecimiento. Finalmente, el artículo presenta también un resumen de las principales bases de datos que podrían servir para realizar análisis sobre el tema en México, y presenta algunas de las más recientes dirigidas a enriquecer los futuros estudios sobre la materia.

Como consecuencia de que la mortalidad ha caído radicalmente, la población ahora vive más tiempo que las generaciones anteriores, aunque esto no significa necesariamente una vida más saludable y una me- 
jor calidad de vida (las discapacidades mentales o físicas:juegan un papel fundamental en ésta). La respuesta a la pregunta de cuál es la condición de vida de la población en edades medias y avanzadas es importante para el diseño de los programas nacionales de salud, los siste mas familiares de apoyo, y la infraestructura institucional de seguridad social en la forma de pensiones de discapacidad o:jubilación; en el futuro, medir la esperanza de vida en salud será tan importante como medir la esperanza de vida de la población. El artículo de Roberto Ham Chande muestra una manera de usar los datos de encuestas nacionales sobre morbilidad o discapacidad para construir indicadores que nos permitan seguir a través del tiempo los cambios que se van dando en la esperanza de vida activa o en la salud de la población. El trabajo ilustra cómo la metodología demográfica se puede usar en el campo de la salud pública para avanzar nuestras agendas de investigación sobre envejecimiento, y los resultados muestran las disparidades que se dan entre la población según diferentes estratos socioeconómicos.

Relacionado estrechamente con la salud y la calidad de vida de la población de edad avanzada se encuentra el cuidado personal que reciben los individuos cuando sus facultades físicas o mentales ya no les permiten la funcionalidad independiente. Para países como México, donde por razones económicas tanto como culturales la norma es cuidar de la atención del anciano dentro del ámbito familiar en lugar del institucional, el papel que:juega el cuidador de la población envejecida es fundamental. La selección del cuidador tiene repercusiones tanto para éste, de manera individual, como para el conjunto de la sociedad; el reconocimiento de dichos roles puede ayudar a diseñar políticas sociales que faciliten una conducción exitosa de ellos. Si se desea alcanzar un envejecimiento con buena calidad de vida, tanto de los que reciben los cuidados en la vejez como de los que los dan, es necesario entender mejor el fenómeno del cuidador en México. El artículo de Leticia Robles Silva presenta resultados de un estudio etnográfico conducido en un barrio de Guadalajara, Jalisco. Concluye que el cuidador del anciano en México generalmente es una cuidadora, y que los varones:juegan un papel importante en la elección de ésta. La selección de la cuidadora sigue patrones jerárquicos que permiten cumplir con los diversos roles competitivos en su vida: trabajadora, cuidadora de menores, y dependiente en sí. El artículo concluye con una serie de resultados que constituyen hipótesis interesantes para probar en estudios cuantitativos a escala nacional, de tal forma que los resultados puedan ser generalizados; igualmente ilustra la forma en que los estudios etnográficos se complementan con los primeros. 
Siguiendo con el énfasis en la salud y la calidad de vida de la población, el artículo de Verónica Montes de Oca se aboca a los determinantes del acceso institucional de la población de edad avanzada al sistema de seguridad social, cubriendo servicios de salud y pensión por:jubilación. El trabajo presenta un resumen de la estructura institucional del sistema de seguridad social para la población de edad media y avanzada en México y continúa con un análisis estadístico multivariado para examinar los determinantes del acceso a la seguridad social, separando los factores sociodemográficos y económicos del individuo de aquellos que representan la estructura institucional. Los resultados concluyen que los factores estructurales de las instituciones determinan en gran medida el acceso a la seguridad social y recomiendan que, si se trata de incrementar su igualdad, en las políticas públicas deben atender a unos y a otros tipos de factores.

Este artículo también tiene una característica importante: ilustra el estado del análisis estadístico en estudios sociodemográficos en México, y las bases de datos con que se cuenta actualmente para realizar este tipo de estudios. Existen ya múltiples bases de datos y se proponen otras en este mismo volumen que nos deben permitir ahondar en el conocimiento que se genera sobre el envejecimiento en México. En el futuro debemos contar con mayor número de trabajos basados en la población a escala nacional, ya que el costo de producir análisis cuantitativos ha disminuido sustancialmente debido al uso generalizado del análisis de datos por computadoras y con herramientas estadísticas.

Además del apoyo familiar e institucional que pueda recibir la población para solventar sus necesidades en la vejez, se ha planteado que la transformación interna del individuo que envejece puede determinar también la ocurrencia de este proceso con buena calidad de vida. Como sociedad que se plantea esto como meta, resulta importante estudiar la manera en que el anciano vive el envejecimiento. Los modelos clásicos de comportamiento demográfico pueden ser inadecuados para estudiar al individuo de edad avanzada porque conforme éste envejece, transforma sus expectativas y su horizonte de planeación, así como sus recursos materiales y su red familiar. El trabajo de Felipe Vázquez Palacios ilustra una manera en que se pueden dar estos cambios en los individuos en un estudio etnográfico, usando una muestra de personas en una agrupación religiosa evangélica en Veracruz, muestra cómo la vida cotidiana durante el periodo de la vejez se encuentra dominada por la espiritualidad. Aunque los resultados obviamente no pueden generalizarse a la población de México, el artículo 
ilustra la manera en que se pueden estudiar los cambios en los individuos y permite formular hipótesis interesantes que podrían ayudar a explicar el comportamiento de la población de edades avanzadas hacia los programas sociales o de salud, el trabajo y el retiro, o hacia la utilización de servicios médicos, entre otros. La investigación hace referencia a variables poco usadas en estudios sociodemográficos del comportamiento humano, por ejemplo, la religión, la espiritualidad, y la fe en general como capital social del que hacen uso las personas para solventar sus necesidades o limitaciones en la vejez, considerando dentro de éstas a la salud, y también a aspectos poco estudiados como el dolor y la soledad. Asimismo, el trabajo ilustra cómo los enfoques cualitativos pueden beneficiar a los futuros estudios sociodemográficos cuantitativos del envejecimiento en México.

Una de las preocupaciones más importantes ante el inminente envejecimiento poblacional se refiere a las fuentes de ingreso con que puede contar la población para solventar sus necesidades económicas en la última etapa de vida cuando su capacidad productiva es poca o nula. En las sociedades en las que la población tiene acceso limitado a los mercados financieros y donde los planes de pensiones son escasos, este aspecto cobra gran importancia. El trabajo de Maria Cristina Gomes da Conceiçáo examina las fuentes de ingreso de los individuos y su posición en el hogar usando bases de datos a escala nacional. Permite contrastar las desigualdades en la población de México y lo compara con Brasil. Este enfoque comparativo enriquece el análisis ya que se trata de dos países con formas y coberturas muy dis. tintas de seguridad social. Por ello, los resultados encuentran una gran diferencia en la dependencia sobre la actividad laboral, de pensiones, y de transferencias informales entre generaciones. Asimismo, los resultados arrojan similitudes entre los dos países respecto a la desigualdad, que se acentúa conforme la edad de los individuos aumenta; esto puede indicar que hay patrones que se cumplen independientemente del sistema estructural del que se trate. Este trabajo, basado en el análisis descriptivo comparativo del nivel, la distribución y los tipos de ingreso, y que usa indicadores no tradicionales de posición en el hogar, ilustra la manera en que se pueden formular y probar importantes hipótesis para un mejor entendimiento de los determinantes de la desigualdad en México y Latinoamérica conforme avance el envejecimiento. En el futuro y conforme se cuente con datos, sería interesante reelaborar parte de este análisis con datos sobre los bienes con que cuentan los individuos: los bienes acumulados de las perso- 
aas de edad avanzada pueden reflejar mejor que los ingresos las relaciones de poder que se dan dentro de los hogares.

Esperamos que el grupo de trabajos presentado en este volumen, con énfasis central en la sociodemografía de la salud durante la edad avanzada, contribuya al estudio y a la toma de decisiones que apunten a mejorar el bienestar de la población ante el envejecimiento poblacional; creemos que este volumen temático sugiere vías teórico-metodológicas renovadas y áreas fértiles de estudio. Si bien nuestra disciplina habitualmente maneja grandes bases de datos y trabaja con encuestas de hogares que posibilitan análisis transversales, algunos de los trabajos aquí presentados ofrecen opciones para integrar estudios longitudinales y de propósitos múltiples a las agendas de investigación acerca de la salud; estos trabajos proponen asumir el reto de profundizar sobre la dinámica de la salud y la discapacidad apoyándose en el conocimien to teórico y metodológico del ciclo de vida y de las transiciones a diferentes estados.

Otra vertiente que se apunta es el aprovechamiento de la experiencia en el manejo de modelos de comportamiento que han servido anteriormente para estudiar con cierto éxito aspectos de la fecundidad y la migración. Se sugiere, por ejemplo, que la experiencia acumulada sobre modelos de distribución del tiempo de las mujeres hacia el trabajo o el cuidado de los niños, sirva también para estudiar la dinámica de atención al anciano; ya se han hecho algunas adaptaciones para contribuir a entender las actitudes de la población en edad avanzada hacia la atención de la salud diferenciando las limitantes que impone el acceso al sistema de servicios y el nivel socioeconómico.

Finalmente, se propone aprovechar la experiencia del sociodemógrafo en el diseño de programas de políticas públicas en el ámbito nacional para aumentar la conciencia hacia los problemas de población. Se sugiere que si en décadas anteriores los mensajes acerca de "las familias pequeñas" fueron usados como instrumentos para enfrentar problemas asociados a las altas tasas de fecundidad, en la actualidad el diseño de programas similares podría contribuir a aumentar la solidaridad generacional con el fin de que las generaciones :jóvenes entiendan la necesidad de aportar al bienestar del anciano mediante la participación en el ámbito público -a través del fisco o apoyando nuevas leyes sobre cuidados a la familia extensa-, y privado, a través del apoyo familiar y personal.

Como lo remarcó Albert Hermalin (1999), las ciencias de la salud y la demografía están muy bien situadas para responder al reto cientí- 
fico que implica la velocidad proyectada y la naturaleza cambiante del envejecimiento poblacional en las próximas décadas. Aunque en este sentido ya se han hecho esfuerzos importantes en México, persiste la necesidad de seguir siendo flexibles e imaginativos, y de coordinar esfuerzos para involucrar a otras disciplinas. Como académicos, debemos contribuir tanto al avance del conocimiento científico con nuevas metodologías y marcos teóricos, como al diseño de políticas públicas.

\section{Bibliografía}

Conapo (1999), Envejecimiento demográfico de México: Retos y perspectivas. México, Consejo Nacional de Población.

Giddens, Anthony (1990), The Consequences of Modernity, Stanford, Calif., Stanford University Press.

Hermalin, Albert (1999), "Setting the Research Agenda on Aging in Latin America: Lessons from Asia", en Kenneth Hill, José Morelos y Rebeca Wong (coords.), Las consecuencias de las transiciones demográfica y epidemiológica en América Latina, México, El Colegio de México.

Hill, Kenneth, José Morelos y Rebeca Wong (coords.) (1999), Las consecuencias de las transiciones demográfica y epidemiológica en América Latina, México, El Colegio de México.

Kinsella, Kevin (2000), "Demographic Dimensions of Global Aging," Journal of Family Issues, vol. 21, núm. 5, pp. 541-558. 